PROCEEDINGS OF THE

AMERICAN MATHEMATICAL SOCIETY

Volume 137, Number 12, December 2009, Pages 3999-4006

S 0002-9939(09)09996-1

Article electronically published on July 24, 2009

\title{
ON THE SYZYGIES OF QUASI-COMPLETE INTERSECTION SPACE CURVES
}

\author{
YOUNGOOK CHOI
}

(Communicated by Bernd Ulrich)

\begin{abstract}
In this paper, we discuss minimal free resolutions of the homogeneous ideals of quasi-complete intersection space curves. We show that if $X$ is a quasi-complete intersection curve in $\mathbb{P}^{3}$, then $I_{X}$ has a minimal free resolution

$$
0 \rightarrow \oplus_{i=1}^{\mu-3} S\left(d_{i+3}+c_{1}\right) \rightarrow \oplus_{i=1}^{2 \mu-4} S\left(-e_{i}\right) \rightarrow \oplus_{i=1}^{\mu} S\left(-d_{i}\right) \rightarrow I_{X} \rightarrow 0,
$$

where $d_{i}, e_{i} \in \mathbb{Z}$ and $c_{1}=-d_{1}-d_{2}-d_{3}$. Therefore the ranks of the first and the second syzygy modules are determined by the number of elements in a minimal generating set of $I_{X}$. Also we give a relation for the degrees of syzygy modules of $I_{X}$. Using this theorem, one can construct a smooth quasicomplete intersection curve $X$ such that the number of minimal generators of $I_{X}$ is $t$ for any given positive integer $t \in \mathbb{Z}^{+}$.
\end{abstract}

\section{Preliminaries}

Let $X$ be a nondegenerate locally Cohen-Macaulay irreducible curve in $\mathbb{P}^{3}$, defined over an algebraically closed field $K$ of characteristic 0 . A curve $X \subset \mathbb{P}^{3}$ is said to be a monomial curve if it has a parametric representation of the form $\left(t_{0}^{n_{3}}, t_{0}^{n_{3}-n_{1}} t_{1}^{n_{1}}, t_{0}^{n_{3}-n_{2}} t_{1}^{n_{2}}, t_{1}^{n_{3}}\right)$ where $n_{1}<n_{2}<n_{3} \in \mathbb{Z}$ with g.c.d. $\left(n_{1}, n_{2}, n_{3}\right)=1$. Let $I_{X} \subset S=K\left[x_{0}, x_{1}, x_{2}, x_{3}\right]$ be the homogeneous ideal of a monomial curve in $\mathbb{P}^{3}$ and let $\mu\left(I_{X}\right)$ be the number of elements in a minimal generating set of $I_{X}$. In his paper ([2]), Bresinsky proves that if $\mu\left(I_{X}\right)=\mu \geq 3$, then $I_{X}$ has the following minimal free resolution:

$$
0 \rightarrow \sum_{j=1}^{\mu-3} S\left(-d_{3, j}\right) \rightarrow \sum_{j=1}^{2 \mu-4} S\left(-d_{2, j}\right) \rightarrow \sum_{j=1}^{\mu} S\left(-d_{1, j}\right) \rightarrow I_{X} \rightarrow 0,
$$

where $d_{i, j} \in \mathbb{Z}$. Therefore the ranks of the first and the second syzygy modules of the homogeneous ideals of monomial curves in $\mathbb{P}^{3}$ are determined by the number of elements in a minimal generating set of $I_{X}$. In this paper, we give a similar result for quasi-complete intersection space curves. Here, a curve $X \subset \mathbb{P}^{3}$ is a

Received by the editors July 1, 2008, and, in revised form, April 5, 2009.

2000 Mathematics Subject Classification. Primary 14M07, 14N05, 14M06.

Key words and phrases. Quasi-complete intersection, monomial curve, minimal free resolution, rank 2 vector bundle, cohomology module.

This work was supported by a Korea Research Foundation Grant funded by the Korean Government (MOEHRD, Basic Research Promotion Fund), KRF-2007-521-C00002.

(C)2009 American Mathematical Society Reverts to public domain 28 years from publication 
quasi-complete intersection if it is locally Cohen-Macaulay and is cut out schemetheoretically by three hypersurfaces in $\mathbb{P}^{3}$. In other words, the sheaf of the ideal $\mathcal{I}_{X}$ of a quasi-complete intersection space curve $X$ has an exact sequence

$$
0 \rightarrow \mathcal{E} \rightarrow \oplus_{i=1}^{3} \mathcal{O}_{\mathbb{P}^{3}}\left(-d_{i}\right) \stackrel{\varphi}{\rightarrow} \mathcal{I}_{X} \rightarrow 0
$$

where $\mathcal{E}$ is a kernel of $\varphi$ and $d_{1}, d_{2}, d_{3}$ are the degrees of the hypersurfaces defin$\operatorname{ing} X$. Note that $\mathcal{E}$ is a rank 2 vector bundle on $\mathbb{P}^{3}$ because $X$ is locally CohenMacaulay. A curve $X$ is called a quasi-complete intersection curve of type $\left(d_{1}, d_{2}, d_{3}\right)$ if $X$ is defined scheme-theoretically by three surfaces $f_{1}, f_{2}, f_{3}$ of degrees $d_{1}, d_{2}, d_{3}$ in $I_{X}$ with $d_{1} \geq d_{2} \geq d_{3}$.

Theorem 1.1. Let $X \subset \mathbb{P}^{3}$ be a quasi-complete intersection space curve of type $\left(d_{1}, d_{2}, d_{3}\right)$ and assume that the homogeneous ideal $I_{X}$ of $X$ has a minimal free resolution

$$
0 \rightarrow L_{2} \rightarrow L_{1} \rightarrow L_{0} \rightarrow I_{X} \rightarrow 0,
$$

where $L_{i}$ is a free $S$-module of rank $\mu_{i}$ for each $i=0,1,2$. Then $\mu_{2}=\mu_{0}-3$ and $\mu_{1}=2 \mu_{0}-4$. Furthermore, $L_{0}=\operatorname{Hom}\left(L_{2}, S\right)(c) \oplus\left(\oplus_{i=1}^{3} S\left(-d_{i}\right)\right)$ and $L_{1} \simeq$ $\operatorname{Hom}\left(L_{1}, S\right)(c)$ where $c=-d_{1}-d_{2}-d_{3}$.

To prove Theorem 1.1, we use results on the first cohomology group of a rank two vector bundle on $\mathbb{P}^{3}$. Let $\mathcal{E}$ be a rank two vector bundle on $\mathbb{P}^{3}$ and let $M_{\mathcal{E}}=H_{*}^{1}(\mathcal{E})$. Assume that $M_{\mathcal{E}}$ has the following minimal free resolution over $S=K\left[x_{0}, x_{1}, x_{2}, x_{3}\right]$ :

$$
0 \rightarrow M_{4} \rightarrow M_{3} \rightarrow M_{2} \rightarrow M_{1} \rightarrow M_{0} \rightarrow M_{\mathcal{E}} \rightarrow 0,
$$

where rank $M_{0}=s$ and rank $M_{1}=t$. Decker and Rao ([5], Proposition 1, and [12, Corollary 2.3) independently show that $t=2 s+2$; i.e., the rank of the first syzygy module is determined by the number of minimal generators of $M_{\mathcal{E}}$. Furthermore Decker gives in his paper ([5]) sufficient conditions for having a cohomology module of a rank 2 vector bundle, i.e., that there exist isomorphisms $\Phi: M_{1}^{*}\left(c_{1}\right) \simeq M_{1}$ and $M_{2} \simeq M_{2}^{\prime} \oplus M_{0}^{*}\left(c_{1}\right)$ such that the composition $M_{0}^{*}\left(c_{1}\right) \stackrel{\sigma}{\rightarrow} M_{1}^{*}\left(c_{1}\right) \stackrel{\Phi}{\simeq} M_{1} \rightarrow M_{0}$ is a trivial morphism. We show in Theorem 1.1 that the morphism $\sigma$ induces a syzygy module morphism $L_{2} \rightarrow L_{1}$. Here, $c_{1}\left(:=c_{1}(\mathcal{E})\right)$ is the first Chern class of a vector bundle $\mathcal{E}$ and $M_{0}^{*}\left(:=\operatorname{Hom}\left(M_{0}, S\right)\right)$ is an $S$-dual module of $M_{0}$.

This paper is organized in the following way: In section 2, we prove Theorem 1.1 and give several corollaries. One of these is the characterization of quasi-complete intersections whose ideals are generated by four homogeneous polynomials. We show that the ideal of $X$ is generated by four elements if and only if the HartshorneRao module $M_{X}$ is a "complete intersection" graded $S$-module with one generator, i.e., $M \simeq S(-d) /\left(f_{1}, f_{2}, f_{3}, f_{4}\right)$ where $f_{1}, f_{2}, f_{3}, f_{4}$ is a regular sequence and $d \in \mathbb{Z}$.

In section 3 , we present a couple of examples and open questions. In particular, for any positive integer $t \in \mathbb{Z}$, one can construct a smooth quasi-complete intersection curve $X$ such that the number of minimal generators of $I_{X}$ is $t$.

Notation. Let $\mu(M)$ be the number of minimal generators of a finitely generated $S$-module $M$. For a curve $X \subset \mathbb{P}^{3}$, we denote the Hartshorne-Rao module by $M_{X}$, i.e., $M_{X}:=H_{*}^{1}\left(\mathcal{I}_{X}\right)$; and for a coherent sheaf $\mathcal{F}$, we write $H_{*}^{i}(\mathcal{F})=\oplus_{k \in \mathbb{Z}} H^{i}(\mathcal{F}(k))$. Also, note that $M^{*}=\operatorname{Hom}(M, S)$ and $M^{\vee}=\oplus_{j \in \mathbb{Z}} \operatorname{Hom}\left([M]_{-j}, K\right)$. 


\section{TheOREM AND COROLLARIES}

To prove Theorem 1.1, we need a nice theorem of Rao.

Theorem 2.1. Let $X$ be a curve in $\mathbb{P}^{3}$ and let $M_{X}$ be its deficiency module, i.e., $M_{X}=H_{*}^{1}\left(\mathcal{I}_{X}\right)$. Assume that $M_{X}$ has a minimal free resolution

$$
0 \rightarrow L_{4} \stackrel{\sigma_{4}}{\rightarrow} L_{3} \rightarrow L_{2} \rightarrow L_{1} \rightarrow L_{0} \rightarrow M_{X} \rightarrow 0 .
$$

Then $I_{X}$ has a minimal free resolution of the form

$$
0 \rightarrow L_{4} \stackrel{\left(\sigma_{4}, 0\right)}{\rightarrow} L_{3} \oplus \oplus_{i=1}^{r} S\left(-l_{i}\right) \rightarrow \oplus_{i=1}^{m} S\left(-e_{i}\right) \rightarrow I_{X} \rightarrow 0
$$

for some integers $e_{i}, l_{i}, r, m$.

Proof. See [13, Theorem 2.5.

Remark 2.2. Note that if $X$ is minimal in its even liaison class with respect to degree, then it can furthermore be shown that the direct summand $\oplus_{i=1}^{r} S\left(-l_{i}\right)$ in Theorem 2.1]does not occur ([10]). One can see in the following proof of Theorem 1.1 that this is also true for a quasi-complete intersection curve $X$ in $\mathbb{P}^{3}$ (Corollary 2.6).

Proof of Theorem 1.1. Since $X$ is a quasi-complete intersection, we have an exact sequence

$$
0 \rightarrow \mathcal{E} \rightarrow \oplus_{i=1}^{3} \mathcal{O}_{\mathbb{P}^{3}}\left(-d_{i}\right) \stackrel{\varphi}{\rightarrow} \mathcal{I}_{X} \rightarrow 0
$$

where $\mathcal{E}$ is a kernel of $\varphi$ which is a rank 2 vector bundle. By the results of ([1], Proposition 1 ) and ([3], Theorem 1.6), the set of scheme-theoretic generators of $X$ always extends to a set of minimal generators of the homogeneous ideal $I_{X}$ of $X$. This set of minimal generators of $I_{X}$ gives a surjective homomorphism $\oplus_{i=1}^{\mu} S\left(-d_{i}\right) \rightarrow I_{X} \rightarrow 0$ where $\mu$ is the number of minimal generators of $I_{X}$. Taking a sheafification, we obtain an exact sequence

$$
0 \rightarrow \mathcal{F} \rightarrow \oplus_{i=1}^{\mu} \mathcal{O}_{X}\left(-d_{i}\right) \rightarrow \mathcal{I}_{X} \rightarrow 0,
$$

with $H_{*}^{1}(\mathcal{F})=0$. Since $X$ is locally Cohen-Macaulay, the coherent sheaf $\mathcal{F}$ is locally free of rank $\mu-1$. These two exact sequences give the following commutative diagram:

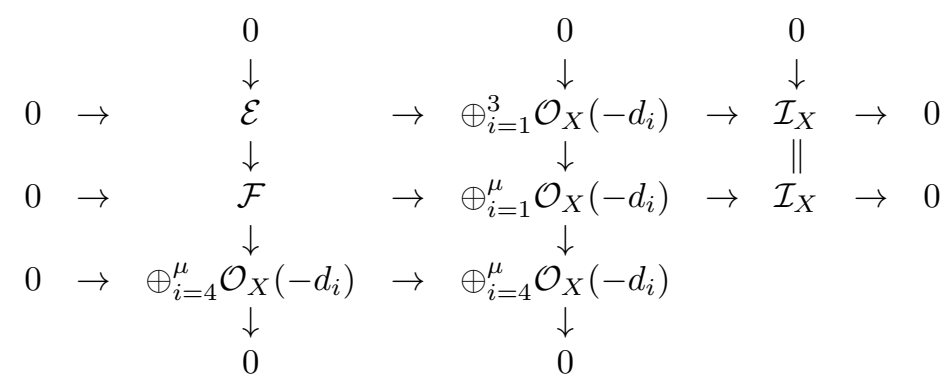

If we take global sections on the first column, we get the exact sequence

$$
0 \rightarrow E \rightarrow H_{*}^{0}(\mathcal{F}) \rightarrow \oplus_{i=4}^{\mu} S\left(-d_{i}\right) \stackrel{\psi}{\rightarrow} M_{\mathcal{E}} \rightarrow 0
$$

where $E=H_{*}^{0}(\mathcal{E})$ and $M_{\mathcal{E}}=H_{*}^{1}(\mathcal{E})$.

By Peterson's theorem ([11], Theorem 6.4.17), $\mu\left(I_{X}\right)-\mu\left(M_{X}^{\vee}\right)=3$ where $M_{X}^{\vee}=$ $\oplus_{j} \operatorname{Hom}\left(\left[H_{*}^{2}(\mathcal{E})\right]_{-j}, K\right)$. Since $M_{X}^{\vee}=\oplus_{j} H^{1}\left(\mathcal{E}\left(-c_{1}-4+j\right)\right)=M_{\mathcal{E}}\left(-c_{1}-4\right)$ and 
$\mu\left(I_{X}\right)=\mu$, we have $\mu\left(M_{\mathcal{E}}\right)=(\mu-3)$. Therefore the morphism $\psi$ is minimal and one has a minimal free resolution,

$$
0 \rightarrow L_{4} \rightarrow L_{3} \rightarrow L_{2} \rightarrow L_{1} \rightarrow \oplus_{i=4}^{\mu} S\left(-d_{i}\right) \rightarrow M_{\mathcal{E}} \rightarrow 0 .
$$

By the theorem of Rao and Decker ([5, 12]), the rank of the free module $L_{1}$ is $2 \mu-4$ and $L_{1}^{*}\left(c_{1}\right) \simeq L_{1}$ where $c_{1}$ is the first Chern class of the vector bundle $\mathcal{E}$. Also, in the exact sequence in ([12], Corollary 2.3), one has a surjection $L_{1}^{*}\left(c_{1}\right) \rightarrow H_{*}^{0}(\mathcal{F}) \rightarrow 0$.

Since $H_{*}^{1}(\mathcal{F})=0$, from the exact sequence in the second row of the above diagram, we have

$$
0 \rightarrow F_{2} \rightarrow L_{1}^{*}\left(c_{1}\right) \stackrel{\phi}{\rightarrow} \oplus_{i=1}^{\mu} S\left(-d_{i}\right) \rightarrow I_{X} \rightarrow 0
$$

where $F_{2}$ is a kernel of the morphism $\phi$. If depth $S / I_{X}=2, F_{2}=0$. Otherwise, depth $S / I_{X}=1$ and the projective dimension of $I_{X}$ is two by the AuslanderBuchsbaum theorem. Therefore $F_{2}$ is a free module. By Serre duality, we have $M_{\mathcal{E}}^{\vee} \simeq M_{X}\left(-c_{1}-4\right) \simeq H_{*}^{2}(\mathcal{E})\left(-c_{1}-4\right) \simeq \operatorname{Ext}_{S}^{4}\left(M_{\mathcal{E}}, S\right)(-4)$, and dualizing the exact sequence (2.1), $M_{X}$ has a minimal free resolution,

$$
0 \rightarrow\left(\oplus_{i=4}^{\mu} S\left(-d_{i}\right)\right)^{*} \rightarrow L_{1}^{*} \rightarrow L_{2}^{*} \rightarrow L_{3}^{*} \rightarrow L_{4}^{*} \rightarrow M_{X}\left(-c_{1}\right) \rightarrow 0 .
$$

By Theorem 2.1 the homogeneous ideal $I_{X}$ of $X$ has a minimal free resolution,

$$
0 \rightarrow\left(\oplus_{i=4}^{\mu} S\left(-d_{i}\right)\right)^{*}\left(c_{1}\right) \rightarrow L_{1}^{*}\left(c_{1}\right) \rightarrow \oplus_{i=1}^{\mu} S\left(-d_{i}\right) \rightarrow I_{X} \rightarrow 0 .
$$

Our first application of Theorem 1.1 is a characterization of quasi-complete intersections whose ideals are generated by four elements. A quasi-complete intersection whose ideal is generated by two elements is a complete intersection, and a quasicomplete intersection whose ideal is generated by three elements is arithmetically Cohen-Macaulay. The geometry of these curves is well-known. Now we characterize quasi-complete intersections whose ideals are generated by four elements.

Lemma 2.3. Assume that $M$ is a "complete intersection" graded $S$-module with one generator, i.e., $M \simeq S\left(-d_{0,1}\right) /\left(f_{1}, f_{2}, f_{3}, f_{4}\right)$ where $f_{1}, f_{2}, f_{3}, f_{4}$ is a regular sequence and $d_{0,1} \in \mathbb{Z}$. Then, $M$ has the minimal free resolution

$$
\begin{aligned}
0 \rightarrow S\left(d_{4,1}\right) \rightarrow \oplus_{i=1}^{4} S\left(-d_{3, i}\right) & \rightarrow \oplus_{i=1}^{6} S\left(-d_{2, i}\right) \\
& \rightarrow \oplus_{i=1}^{4} S\left(-d_{1, i}\right) \rightarrow S\left(-d_{0,1}\right) \stackrel{\psi}{\rightarrow} M \rightarrow 0
\end{aligned}
$$

for some $d_{i, j} \in \mathbb{Z}$.

Proof. This is a Koszul complex and is exact since $f_{1}, f_{2}, f_{3}, f_{4}$ is a regular sequence.

Corollary 2.4. Among all quasi-complete intersections with $\mu(X) \geq 4, \mu(X)=4$ if and only if the Hartshorne-Rao module $M_{X}$ is a "complete intersection" module.

Proof. Assume that $X$ is a quasi-complete intersection of type $\left(d_{1}, d_{2}, d_{3}\right)$ with $\mu(X)=4$. By Theorem 1.1, $I_{X}$ has a minimal free resolution,

$$
0 \rightarrow S\left(d_{4}-d_{1}-d_{2}-d_{3}\right) \rightarrow \oplus_{i=1}^{4} S\left(-e_{i}\right) \rightarrow \oplus_{i=1}^{4} S\left(-d_{i}\right) \rightarrow I_{X} \rightarrow 0,
$$

for some integers $e_{i}$ and $d_{4}$. Then, by Theorem 2.1, the Hartshorne-Rao module $M_{X}$ of $X$ has a minimal free resolution,

$$
0 \rightarrow L_{4} \rightarrow L_{3} \rightarrow L_{2} \rightarrow L_{1} \rightarrow L_{0} \rightarrow M_{X} \rightarrow 0,
$$


with $\operatorname{rank} L_{4}=1$ and $\operatorname{rank} L_{3}=4$. Dualizing the exact sequence, we get

$$
0 \rightarrow L_{0}^{*} \rightarrow L_{1}^{*} \rightarrow L_{2}^{*} \rightarrow L_{3}^{*} \rightarrow L_{4}^{*} \rightarrow \operatorname{Ext}_{S}^{4}\left(M_{X}, S\right) \rightarrow 0 .
$$

This gives that $\operatorname{Ext}_{S}^{4}\left(M_{X}, S\right)$ is a complete intersection module with one generator because $\operatorname{Ext}_{S}^{4}\left(M_{X}, S\right)$ is a finite module. By Lemma 2.3. $\operatorname{rank} L_{0}=1$ and $\operatorname{rank} L_{1}=$ 4 , and it follows that $M_{X}$ is a complete intersection module with one generator.

Assume that $M_{X}$ is a complete intersection module with one generator. Then $M_{X}$ has a minimal free resolution,

$$
0 \rightarrow L_{0}^{*} \rightarrow L_{1}^{*} \rightarrow L_{2}^{*} \rightarrow \oplus_{i=1}^{4} S\left(e_{i}\right) \rightarrow S(-d) \rightarrow M_{X} \rightarrow 0
$$

By Lemma 2.3. $\operatorname{rank} L_{0}=1$, rank $L_{1}=4$ and $\operatorname{rank} L_{2}=6$. By Theorem 1.1,

$$
0 \rightarrow L_{0}^{*} \rightarrow L_{1}^{*} \rightarrow \oplus_{i=1}^{4} S\left(-f_{i}\right) \rightarrow I_{X} \rightarrow 0
$$

for some $f_{i} \in \mathbb{Z}$. Therefore, we get $\mu(X)=4$.

If $X$ is a subcanonical quasi-complete intersection and $M_{X}$ is generated by one element, then $M_{X}$ is a complete intersection module with one generator and by Corollary (2.4), $\mu\left(I_{X}\right)=4$. One can compare this result to the following theorem of Bresinsky, Schenzel, and Vogel.

Theorem 2.5 ([4], Theorem 2). Let $X$ be a Buchsbaum, non-arithmetically CohenMacaulay curve in $\mathbb{P}^{3}$. Let $N=\operatorname{dim} M_{X}$. If $X$ is a quasi-complete intersection, then $\mu\left(I_{X}\right)=4$ and $N=1$.

Also, note that by Rao's theorem ([12, Corollary 3.1) and Decker's theorem ([5], Proposition 1), $\operatorname{Ext}_{S}^{4}\left(M_{X}, S\right)$ in the proof of Corollary 2.4 is a first cohomology module of a rank two vector bundle.

Another application of Theorem 1.1 is that if $X$ is a quasi-complete intersection curve of type $\left(d_{1}, d_{2}, d_{3}\right)$, then one can bound the degree of minimal generators of $I_{X}$ in terms of $\left(d_{1}, d_{2}, d_{3}\right)$. This bound is sharp as the homogeneous ideal of a twisted cubic curve is generated by quadric polynomials.

Corollary 2.6. Let $X$ be a quasi-complete intersection curve of type $\left(d_{1}, d_{2}, d_{3}\right)$ in $\mathbb{P}^{3}$ with deficiency module $M_{X}$. Assume that $M_{X}$ has a minimal free resolution

$$
0 \rightarrow L_{4} \stackrel{\sigma_{4}}{\rightarrow} L_{3} \rightarrow L_{2} \rightarrow L_{1} \rightarrow L_{0} \rightarrow M_{X} \rightarrow 0 .
$$

Then $I_{X}$ has a minimal free resolution of the form

$$
0 \rightarrow L_{4} \stackrel{\sigma_{4}}{\rightarrow} L_{3} \rightarrow L_{4}^{*}\left(c_{1}\right) \oplus\left(\oplus_{i=1}^{3} S\left(-d_{i}\right)\right) \rightarrow I_{X} \rightarrow 0,
$$

where $c_{1}=-\left(d_{1}+d_{2}+d_{3}\right)$.

Proof. This is clear from the proof of Theorem 1.1 and Theorem 2.1

Corollary 2.7. If $X$ is a quasi-complete intersection curve of type $\left(d_{1}, d_{2}, d_{3}\right)$ in $\mathbb{P}^{3}$, then the homogeneous ideal $I_{X}$ is generated by homogeneous polynomials of degree $\leq d_{1}+d_{2}-2$.

Proof. By Theorem 1.1. $I_{X}$ has a minimal free resolution,

$$
0 \rightarrow \oplus_{i=1}^{\mu-3} S\left(d_{i+3}+c_{1}\right) \rightarrow \oplus_{i=1}^{2 \mu-4} S\left(-e_{i}\right) \rightarrow \oplus_{i=1}^{\mu} S\left(-d_{i}\right) \rightarrow I_{X} \rightarrow 0 .
$$

Let $m_{1}=\max \left\{d_{i}\right\}, m_{2}=\min \left\{d_{i}\right\}$ and $n_{1}=\max \left\{e_{i}\right\}$. It is well-known that the minimal degree of the hypersurface containing a quasi-complete intersection $X$ of type $\left(d_{1}, d_{2}, d_{3}\right)$ is $d_{3}$, i.e., $m_{2}=d_{3}([1])$. By ([7], page 304), we have $m_{1}<n_{1}$. On the other hand, we get $n_{1}<-\left(c_{1}+m_{2}\right)$ since the dual of the exact sequence (2.2) 
is also a minimal free resolution of $\operatorname{Ext}_{S}^{4}\left(M_{X}, S\right)$ and the minimal twisting numbers of syzygy modules are increasing. Therefore, we have

$$
m_{1} \leq-c_{1}-d_{3}-2=\left(d_{1}+d_{2}\right)-2 .
$$

Finally, one can consider Theorem 1.1 as a generalization of the following corollary.

Corollary 2.8. If $X$ is a quasi-complete intersection curve in $\mathbb{P}^{3}$ defined by three surfaces $f_{1}, f_{2}, f_{3}$ in $I_{X}$ of degrees $d_{1}, d_{2}, d_{3}$, then we have

$$
M_{X}^{\vee}(4) \simeq \operatorname{Ext}_{S}^{4}\left(M_{X}, S\right) \simeq I_{X} /\left(f_{1}, f_{2}, f_{3}\right)\left(d_{1}+d_{2}+d_{3}\right)
$$

as an $S$-module.

Proof. See ([13], page 215) or (4], page 289).

Corollary 2.8 just gives an isomorphism between two $S$-modules $M_{X}^{\vee}(4)$ and $I_{X} /\left(f_{1}, f_{2}, f_{3}\right)\left(d_{1}+d_{2}+d_{3}\right)$. Theorem 1.1] states that if $X$ is a quasi-complete intersection, then a minimal free resolution of the homogeneous ideal $I_{X}$ is determined by a minimal free resolution of the Hartshorne-Rao module $M_{X}$.

\section{EXAMPLES AND OPEN QUESTIONS}

Example 3.1. Let $X$ be a Buchsbaum curve in $\mathbb{P}^{3}$ with $H^{1}\left(\mathcal{I}_{X}(k)\right) \neq 0$ for some integer $k$. If $X$ is defined scheme-theoretically by three surfaces $f_{1}, f_{2}, f_{3}$ of degrees $d_{1}, d_{2}, d_{3}$ in $I_{X}$, then $\operatorname{dim}\left(M_{X}\right)=1$ and the number of minimal generators of $I_{X}$ is 4 (11]). Therefore, by Theorem 1.1 the homogeneous ideal $I_{X}$ has a minimal free resolution,

$$
0 \rightarrow S\left(d_{4}-d_{1}-d_{2}-d_{3}\right) \rightarrow \oplus_{i=1}^{4} S\left(-e_{i}\right) \rightarrow \oplus_{i=1}^{4} S\left(-d_{i}\right) \rightarrow I_{X} \rightarrow 0,
$$

for some integers $e_{i}$ and $d_{4}$.

Example 3.2. Let $X$ be a smooth elliptic curve in $\mathbb{P}^{3}$ of degree $t+4$. Since $\omega_{X}=\mathcal{O}_{X}, X$ is the zero locus of a section of a rank two vector bundle $\mathcal{F}$ on $\mathbb{P}^{3}$ with $c_{1}(\mathcal{F})=4$ and $c_{2}(\mathcal{F})=t+4([12$, Example 3.5, and [8], (4.3.3)). If $\mathcal{E}=\mathcal{F}(-2)$, we have

$$
0 \rightarrow \mathcal{O}_{\mathbb{P}^{3}}(-4) \rightarrow \mathcal{E}(-2) \rightarrow \mathcal{I}_{X} \rightarrow 0 .
$$

Since $H^{1}\left(\mathcal{P}^{3}, \mathcal{I}_{X}(l)\right)=0$ for $l \leq 0, \mathcal{E}$ is a mathematical instanton bundle. Since $H^{1}(\mathcal{E}(-1))=H^{1}\left(\mathcal{I}_{X}(1)\right)=t, M_{\mathcal{E}}$ has a minimal free resolution,

$$
\oplus S\left(-d_{i}\right) \rightarrow S^{\oplus 2 t+2} \rightarrow S(1)^{\oplus t} \rightarrow M_{\mathcal{E}} \rightarrow 0 .
$$

By Theorem 2.5 in [6], a residual $Y$ of $X$ in a complete intersection of two surfaces of degrees $d_{1}$ and $d_{2}$ is a quasi-complete intersection of type $\left(d_{1}+d_{2}-4, d_{1}, d_{2}\right)$. By Theorem 1.1] the homogeneous ideal $I_{Y}$ has a minimal free resolution,

$$
\begin{aligned}
0 \rightarrow \oplus_{i=1}^{t} S\left(f_{i}+c_{1}\right) & \rightarrow L_{1} \\
& \rightarrow \oplus_{i=1}^{t} S\left(-f_{i}\right) \oplus S\left(-d_{1}\right) \oplus S\left(-d_{2}\right) \oplus S\left(-d_{1}-d_{2}+4\right) \rightarrow I_{Y} \rightarrow 0,
\end{aligned}
$$

where $c_{1}=-\left(2 d_{1}+2 d_{2}-4\right)$ and $f_{i} \in \mathbb{Z}$. Therefore, for any given number $t$, one can construct a quasi-complete intersection curve $Y$ in $\mathbb{P}^{3}$ such that the number of minimal generators of $I_{Y}$ is $t$.

Prof. Schenzel raises the following questions: 
Question 3.3. Bresinsky's work ([2]) and Theorem 1.1] determine the structure of the minimal free resolutions of homogeneous ideals $I_{X}$ of monomial curves and quasi-complete intersections. What is the common generalization of monomial curves and quasi-complete intersections? How do we enlarge the classes of varieties which have the same type of minimal free resolutions as in Theorem 1.1]

For example, one of the nice properties of monomial curves is the following:

Theorem 3.4 (4, Theorem 3). Let $X$ be a monomial curve in $\mathbb{P}^{3}$ over an algebraically closed field $K$. Then the following conditions are equivalent:

(1) $\mu\left(I_{X}\right)=4$ and $X$ lies on a quadric.

(2) There is an integer $n \geq 1$ such that the minimal free resolution of $S / I_{X}$ has the form

$$
0 \rightarrow S(-2 n-3) \rightarrow S^{4}(-2 n-2) \rightarrow S(-2) \oplus S^{3}(-2 n-1) \rightarrow S \rightarrow S / I_{X} \rightarrow 0 .
$$

On the other hand, by Corollary 2.4, if $X$ is a quasi-complete intersection of type $\left(d_{1}, d_{2}, d_{3}\right)$ with $\mu\left(I_{X}\right)=4$, then $I_{X}$ has the following minimal free resolution:

$$
0 \rightarrow S\left(d_{4}-d_{1}-d_{2}-d_{3}\right) \rightarrow \oplus_{i=1}^{4} S\left(-e_{i}\right) \rightarrow \oplus_{i=1}^{4} S\left(-d_{i}\right) \rightarrow I_{X} \rightarrow 0
$$

for some integers $e_{i}$ and $d_{4}$.

Therefore, it would also be very interesting to know whether there exists a monomial curve $X$ that has $\mu\left(I_{X}\right)=4$ but is not a quasi-complete intersection.

Question 3.5. How can we characterize the monomial curves in $\mathbb{P}^{3}$ that are quasicomplete intersections?

\section{ACKNOWLEDGEMENT}

The author would like to thank Prof. P. Schenzel for many valuable comments on this paper.

\section{REFERENCES}

[1] V. Beorchia, Ph. Ellia, On the equations defining quasicomplete intersection space curves, Arch. Math. (Basel) 70 (1998) 244-249. MR1604080 (98k:14042)

[2] H. Bresinsky, Minimal free resolutions of monomial curves in $\mathbb{P}^{3}$, Linear Alg. Appl. 59 (1984) 121-129. MR743050 (85d:14042)

[3] H. Bresinsky, P. Schenzel, J. Stückrad, Quasi-complete intersection ideals of height 2, J. Pure Appl. Algebra 127 (1998) 137-145. MR.1620704 (99d:13014)

[4] H. Bresinsky, P. Schenzel, W. Vogel, On liaison, arithmetical Buchsbaum curves and monomial curves in $\mathbb{P}^{3}$, J. Algebra 86 (1984) 283-301. MR732252 (85c:14031)

[5] W. Decker, Monads and cohomology modules of rank 2 vector bundles, Compositio Math. 76 (1990) 7-17. MR:1078855 (91k:14030)

[6] D. Franco, S. Kleiman, A. Lascu, Gherardelli linkage and complete intersections, Michigan Math. J. 48 (2000) 271-279. MR1786490(2002a:14057)

[7] M. Green, R. Lazarsfeld, Some results on the syzygies of finite sets and algebraic curves, Compositio Math. 67 (1988) 301-314. MR.959214 (90d:14034)

[8] R. Hartshorne, Stable vector bundles of rank 2 on $\mathbb{P}^{3}$, Math. Ann. 238 (1978) 229-280. MR514430 (80c:14011)

[9] F. Horrocks, Vector bundles on the punctured spectrum of a local ring, Proc. London Math. Soc. 14 (3) (1964) 689-713. MR0169877 (30:120)

[10] M. Martin-Deschamps, D. Perrin, Sur la classification des courbes gauches, Astérisque 184185 (1990). MR:1073438 (91h:14039)

[11] J. Migliore, Introduction to Liaison Theory and Deficiency Modules, Birkhäuser Boston, 1998. MR:1712469 (2000g:14058) 
[12] P. Rao, A note on cohomology modules of rank two bundles, J. Algebra 86 (1984) 23-34. MR:727366 (85b:14022)

[13] P. Rao, Liaison among curves in $\mathbb{P}^{3}$, Invent. Math. $50(1978 / 79)$ 205-217. MR520926 (80e:14023)

Department of Mathematics Education, Yeungnam University, 214-1 Daedong Gyeongsan, 712-749, Gyeongsangbuk-Do, Republic of Korea

E-mail address: ychoi824@ynu.ac.kr 